\title{
Accuracy of Strain in Strain Maps Improved by Averaging Multiple Maps
}

\author{
Noriaki Endo and Yukihito Kondo
}

EM Business Unit, JEOL Ltd., 3-1-2 Musashino, Akishima, Tokyo 196-8558, Japan.

Several methods have been proposed and tried for strain analysis of a crystal on nano-scale. Diffractometry such as nano-beam diffraction [1] and convergent-beam electron diffraction [2][3], and the phase imaging methods such as dark-filed electron holography [4] and high-resolution electron microscopy [5] have been applied to this issue. In scanning transmission electron microscopy (STEM), when the raster spacing is close to the one of a crystal lattice or the multiple of it and the probe size is smaller than the crystal lattice spacing, we sometimes observe the moiré fringes due to the under-sampling effect. We applied the fringe to detect strain in practical semiconductor devices and succeeded to map and quantify the strain [6]. However, the required accuracy of the strain measurement in the semiconductor industry is as high as or better than $0.2 \%$. In this paper, we report how we improve the accuracy of the strain measurement by using multiple strain maps.

The microscopes we used were JEM-ARM200F and JEM-2800. A lamella of a practical pMOS silicon device was prepared with an FIB. The thickness of this lamella was measured to be approximately 210 $\mathrm{nm}$ with the electron energy-loss spectroscopy. The strain analysis procedure with the STEM moiré method was performed as the previous report [6].

To measure the accuracy of this method, we performed the strain analysis on a non-strained Si single crystal. Figure 1 (a) shows HAADF-STEM moiré image at the right and its strain map at the left. The STEM moiré fringe in Figure 1 (a) was obtained from the lattice fringes between Si [111] and the scanning raster. We took 30 STEM images with the moiré fringes in the same field of view, and reconstructed each averaged strain map from these STEM images. The raw strain map is generally affected by slow and slight displacement of the sample during image acquisition. To eliminate this influence, we tried to use a rectangle region that covers all vertical pixels as a reference, since the region includes the information of the specimen displacement. The displacement manifests itself as a strain in the strain map. The amount of strain due to the displacement always appears as a vertical change of strain. Therefore, the more accurate strain map can be obtained by subtracting the averaged strain over the reference map from the raw map. The accuracy of the lattice strain is shown as the standard deviation $(\sigma)$ of the lattice strain, which was measured in the whole field of view, since the non-strained crystal ideally shows constant strain anywhere in the map. Figure 1 (b) plots the standard deviation of strain with respect to the number of STEM moiré images to make the averaged strain maps. From the plots, the 20 or more images are necessary to satisfy the required accuracy of $\pm 0.2 \%$ for the strain measurement in the semiconductor industry.

Figure 2 shows the HAADF-STEM moiré image of the silicon device (a), and its strain map (b). The STEM moiré fringe in Figure 2 (a) was obtained from the lattice fringes between Si [220] and the scanning raster. In Figure 2 (b), the subtracted strain map by a reference region is shown. The reference region was set in the right region of the field of view, since the region is considered to be non-strained. Figure 2 (b) is the stain map averaged 20 strain maps. The amount of strain under the gate was measured as a compressive strain of approximately $-1.9 \%$ from the profile of the amount of strain in Figure 2 (c). 
By averaging, the strain distribution of a crystal can reproducibly be measured from only a low-magnification STEM image in a wide field which is useful for the characterization of the strained devices with the accuracy of strain better than $0.2 \%$.

\section{References:}

[1] K. Usuda et al., Mat. Sci. Eng. B 124 (2005), p.143.

[2] K. Tsuda et al., J. Electron. Microsc. 56(2) (2007), p.57.

[3] S. Kramer et al., Ultramicroscopy 81(3-4) (2000), p.245.

[4] M.J. Hytch et al., Nature 453(2008), p.1086.

[5] M.J. Hytch et al., Ultramicroscopy 74 (1998), p.131.

[6] N. Endo and Y. Kondo, Microsc. Microanal. 19 (suppl.2) (2013), p.346.

(a)

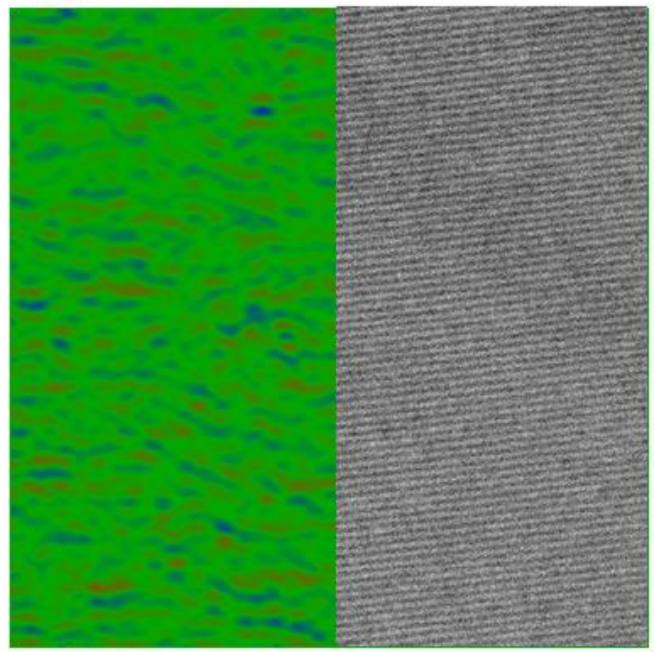

(b)

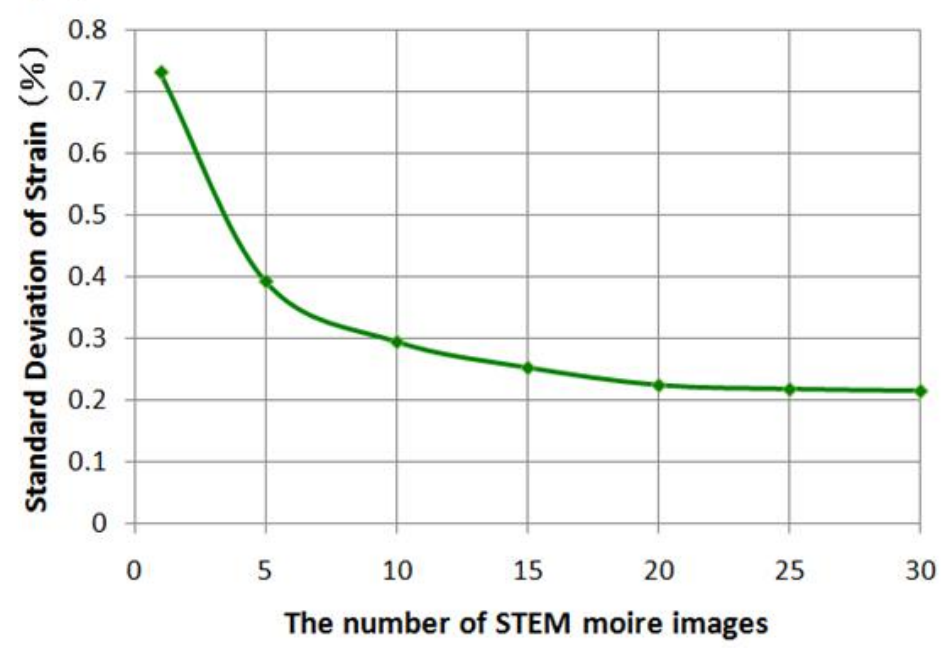

Figure. 1. Strain map (left) reconstructed from STEM moiré fringes (right) of a Si single crystal with no strain (a) and relation between the number of STEM moiré images to make an averaged strain map and the standard deviation of strain of the averaged strain maps (b).

(a)

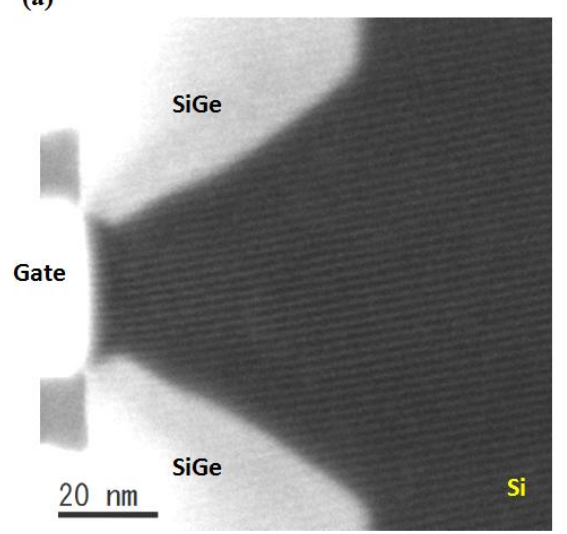

(b)

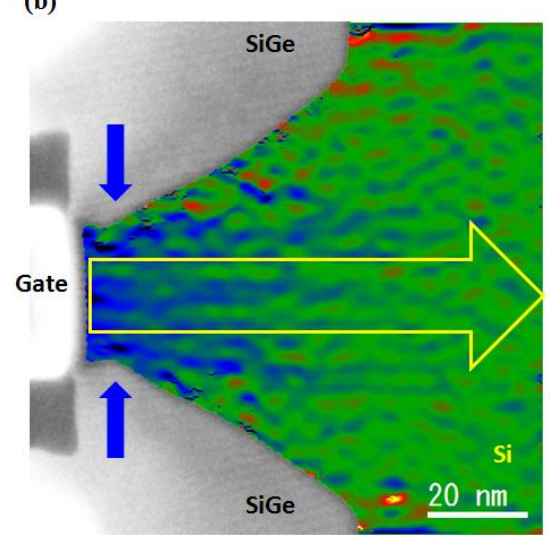

(c)

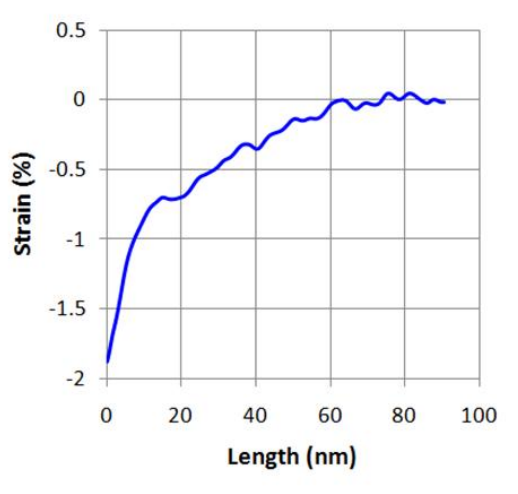

Figure. 2. Strain map (b) reconstructed from STEM moiré fringes (a) of a semiconductor device and the profile of the amount of strain (c) along the arrow of this strain map. 